\title{
Distribuição e autocorrelação espacial dos casos de Tentativas de Suicídio por medicamentos em um município paraibano
}

\author{
Distribution and spatial self-correlation of cases of Suicide Attempts by drug in a municipality in \\ paraiban
}

Distribución y autorrelación espacial de casos de Intento de Suicidio por droga en un municipio de paraibano

Recebido: 08/11/2021 | Revisado: 17/11/2021 | Aceito: 21/11/2021 | Publicado: 01/12/2021

\author{
Andreza Josiany Aires de Farias \\ ORCID: https://orcid.org/0000-0001-6631-9186 \\ Universidade Estadual da Paraíba, Brasil \\ E-mail: ndrezaafarias@gmail.com \\ Allana Renally Cavalcante Santos de Moraes \\ ORCID: https://orcid.org/0000-0002-6753-8687 \\ Universidade Estadual da Paraíba, Brasil \\ E-mail: allanarenally@gmail.com \\ Ricardo Alves de Olinda \\ ORCID: https://orcid.org/0000-0002-0509-8428 \\ Universidade Estadual da Paraíba, Brasil \\ E-mail: ricardo.estat@yahoo.com.br \\ Saulo Rios Mariz \\ ORCID: https://orcid.org/0000-0001-7988-9516 \\ Universidade Federal de Campina Grande, Brasil \\ E-mail: sjmariz22@hotmail.com \\ Tânia Maria Ribeiro Monteiro de Figueiredo \\ ORCID: https://orcid.org/0000-0001-6197-2936 \\ Universidade Estadual da Paraíba, Brasil \\ E-mail: taniaribeiro@ccbs.uepb.edu.br \\ Sayonara Maria Lia Fook \\ ORCID: https://orcid.org/0000-0003-1948-9371 \\ Universidade Estadual da Paraíba, Brasil \\ E-mail: sayonara fook@ servidor.uepb.edu.br
}

\begin{abstract}
Resumo
Trata-se de um estudo observacional, analítico e transversal, de caráter quantitativo, realizado a partir de dados secundários provenientes do Sistema de Informação de Agravos de Notificação (SINAN). Objetivou-se analisar o padrão espacial dos casos de tentativas de suicídio por medicamentos no município de Campina Grande/PB, em 2019. Foi realizado estatística descritiva, assim como elaboração de mapas (Moran e Lisa) para a verificação da distribuição espacial dos casos em estudo. Realizou-se, também, mapa coroplético para demonstrar a ocorrência e a intensidade do fenômeno e Diagrama de Espalhamento de Moran, utilizando-se o Índice de Moran Global e o Índice de Moran Local, com auxílio do software estatístico gratuito The R Project for Statistical Computing. Para nortear a análise espacial, utilizou-se a variável "bairro". A amostra totalizou 237 pacientes residentes na zona urbana de Campina Grande que tentaram suicídio através do uso de medicamentos. O perfil dos pacientes obedeceu o seguinte padrão: sexo feminino (79,7\%), faixa etária de 20 a 39 anos (51,5\%), escolaridade em nível fundamental $(49,4 \%)$, solteiro (a) como estado civil $(64,5 \%)$ e a maioria sendo estudante $(42,2 \%)$, seguido de desempregado (a) $(32,1 \%)$. Os bairros que apresentaram o maior número de casos foram: Malvinas $(n=23)$, Bodocongó $(n=21)$ e Serrotão $(n=9)$. Por outro lado, os bairros Mirante, Cuités, Sandra Cavalcante, Louzeiro, Itararé e Velame não apresentaram ocorrências de casos de tentativas de suicídio através do uso de medicamentos. Assim, verificou-se que, no quesito global, não houve autocorrelação espacial significativa na região estudada, com Índice de Moran Global encontrado de 0,1210.
\end{abstract}

Palavras-chave: Análise espacial; Suicídio; Saúde pública.

\begin{abstract}
This is an observational, analytical and cross-sectional study, with a quantitative character, carried out from secondary data from the Notifiable Diseases Information System (SINAN). The objective was to analyze the spatial pattern of cases of suicide attempts by medication in the city of Campina Grande/PB, in 2019. Descriptive statistics were performed, as well as the elaboration of maps (Moran and Lisa) to verify the spatial distribution of cases in study. A choropleth map was also carried out to demonstrate the occurrence and intensity of the phenomenon and a Moran
\end{abstract}


Scattering Diagram, using the Global Moran Index and the Local Moran Index, with the help of the free statistical software The R Project for Statistical Computing. To guide the spatial analysis, the "neighborhood" variable was used. The sample totaled 237 patients residing in the urban area of Campina Grande who attempted suicide through the use of medication. The profile of the patients followed the following pattern: female (79.7\%), aged from 20 to 39 years $(51.5 \%)$, education at elementary level $(49.4 \%)$, single with marital status $(64.5 \%)$ and the majority being students (42.2\%), followed by unemployed (32.1\%). The districts with the highest number of cases were: Malvinas $(n=23)$, Bodocongó $(n=21)$ and Serrotão $(n=9)$. On the other hand, the neighborhoods Mirante, Cuités, Sandra Cavalcante, Louzeiro, Itararé and Velame did not present cases of suicide attempts through the use of medication. Thus, it was found that, in the global aspect, there was no significant spatial autocorrelation in the studied region, with a Global Moran Index found of 0.1210 .

Keywords: Spatial analysis; Suicide; Public health.

\section{Resumen}

Se trata de un estudio observacional, analítico y transversal, de carácter cuantitativo, realizado a partir de datos secundarios del Sistema de Información de Enfermedades Notificables (SINAN). El objetivo fue analizar el patrón espacial de casos de intentos de suicidio por medicación en la ciudad de Campina Grande / PB, en 2019. Se realizó estadística descriptiva, así como la elaboración de mapas (Moran y Lisa) para verificar la distribución espacial de casos en estudio. También se realizó un mapa de coropletas para demostrar la ocurrencia e intensidad del fenómeno y un Diagrama de Dispersión de Moran, utilizando el Índice de Moran Global y el Índice de Moran Local, con la ayuda del software estadístico gratuito The R Project for Statistical Computing. Para orientar el análisis espacial se utilizó la variable "vecindad". La muestra fue de 237 pacientes residentes en el área urbana de Campina Grande que intentaron suicidarse mediante el uso de medicamentos. El perfil de los pacientes siguió el siguiente patrón: femenino (79,7\%), de 20 a 39 años $(51,5 \%)$, educación básica (49,4\%), soltero en estado civil $(64,5 \%)$ y mayoría estudiantes (42,2\%), seguido de los desempleados $(32,1 \%)$. Los distritos con mayor número de casos fueron: Malvinas ( $\mathrm{n}=23$ ), Bodocongó $(\mathrm{n}=21)$ y Serrotão $(\mathrm{n}=9)$. Por otro lado, los barrios Mirante, Cuités, Sandra Cavalcante, Louzeiro, Itararé y Velame no tuvieron casos de intentos de suicidio por uso de medicamentos. Así, se encontró que, en el aspecto global, no hubo autocorrelación espacial significativa en la región estudiada, encontrándose un Índice de Moran Global de 0,1210.

Palabras clave: Análisis espacial; Suicidio; Salud pública.

\section{Introdução}

A intoxicação exógena é causada pelo contato com substâncias químicas com poder de provocar prejuízos à saúde do indivíduo, inclusive a morte (Vieira, Santana \& Suchara, 2015). Klinger et al. (2016) apontam que ela pode ser ocasionada de forma acidental ou proposital.

Dessa forma, para Mota et al. (2012), o medicamento tem sido considerado um dos principais agentes responsáveis por intoxicações que acarretam na hospitalização em emergências e, de forma mais grave, em óbito. O uso irracional de medicamentos tem se mostrado como um dos graves problemas de saúde pública do Brasil e do mundo (Who, 2019). Neste sentido, entende-se que o avanço da indústria farmacêutica, a maior disponibilidade desses recursos e a consequente banalização da automedicação são alguns dos fatores que têm contribuído para o aumento dos casos de intoxicação por medicamentos em idades diversas, ambos os sexos e diferentes situações socioeconômicas (Maior, Osorio-de-Castro \& Andrade, 2017).

Assim sendo, as intoxicações estão incluídas na Lista Nacional de Notificação Compulsória de Doenças, Agravos e Eventos de Saúde Pública e passaram a ser de notificação compulsória através da publicação da Portaria $\mathrm{n}^{\circ} 104$, de 25 de janeiro de 2011 (Brasil, 2011). Esta Portaria foi revogada pela Portaria no 1271, de 6 de junho de 2014, que torna imediata (em até 24 horas) a notificação de casos de Tentativas de Suicídio (TS) na esfera municipal, com objetivo de garantir intervenção imediata dos casos (Brasil, 2014).

Dessa maneira, a tentativa de suicídio é considerada a principal circunstância dessas intoxicações (Timotéo et al., 2020), uma vez que caracteriza-se pelo ato proposital de autoagressão que visa a morte, porém, não tem sucesso (Félix et al., 2016).

O Brasil registrou, em 2019, no Sistema de Informação de Agravos de Notificação (SINAN) 177.357 casos de 
intoxicação exógena, sendo 85.178 casos resultados de tentativa de suicídio. A Paraíba, por sua vez, notificou, no mesmo ano, 3.657 casos de intoxicação exógena, sendo 1.168 casos de tentativa de suicídio, alcançando o primeiro lugar das circunstâncias de intoxicação (Sinan, 2021).

A cidade de Campina Grande também acompanha o perfil que abrange o país e o Estado da Paraíba e, de forma parecida, também apresentou alta nos casos de intoxicação exógena (707 casos), sendo 390 atribuídos às tentativas de suicídio, dos quais 290 casos $(74,4 \%)$ eram do sexo feminino e 100 casos $(25,6 \%)$ relacionados ao sexo masculino.

Os fatores que contribuem para essa problemática incluem aspectos epidemiológicos e pessoais, como gênero, faixa etária, escolaridade, situação no mercado de trabalho, assim como questões emocionais, comportamentais e cognitivos. Essas informações tonam-se de grande importância para orientar o cuidado que deve ser voltado para essas pessoas, com atuação na prevenção de novos agravos (Moreira, Martins, do Amaral Gubert \& Sousa, 2015).

Diante do quadro epidemiológico apresentado, percebe-se a necessidade de inclusão de novos estudos e tecnologias a fim de incrementar ações voltadas ao planejamento de ações de prevenção de agravos relacionados às intoxicações exógenas e promoção da saúde para a população.

Dessa forma, a Análise Espacial é uma importante ferramenta que permite realizar o diagnóstico situacional de determinada região e possibilita a avaliação dos resultados de acordo com a realidade socioeconômica, cultural, ambiental (Magalhães \& Medronho, 2017).

Diante do exposto, o estudo propõe analisar o padrão espacial dos casos de tentativas de suicídio por medicamentos no município de Campina Grande/PB, em 2019.

\section{Metodologia}

Trata-se de um estudo observacional, analítico e transversal, de caráter quantitativo. Pereira et al. (2018) afirma que o estudo transversal permite a obtenção de informações fidedignas ao final da pesquisa, ao passo que elabora conclusões confiáveis e gera hipóteses que servem de investigação para outras pesquisas futuras.

A presente pesquisa foi realizada a partir de dados secundários provenientes do Sistema de Informação de Agravos de Notificação (SINAN) referentes ao ano de 2019, no município de Campina Grande, Paraíba, Brasil.

A cidade de Campina Grande é a segunda cidade mais populosa da Paraíba, com população aproximada de 410 mil habitantes e está localizada na mesorregião do Agreste Paraibano. A coleta de dados foi realizada de forma manual através do banco de dados do CIATox-CG, localizado no Hospital de Emergência e Trauma Dom Luiz Gonzaga Fernandes, na cidade de Campina Grande, onde são realizadas as notificações dos pacientes vítimas de intoxicação exógena, assim como acidentes por animais peçonhentos. A população estudada engloba os casos de Tentativa de Suicídio através do uso de medicamentos, notificados em 2019.

A população foi caracterizada considerando-se as variáveis sociodemográficas: sexo, faixa etária, estado civil, nível de escolaridade e situação no mercado de trabalho.

Os dados foram coletados com auxílio da Ficha de Investigação de Intoxicação utilizada pelo Sistema Nacional de Agravos de Notificação (SINAN) para notificação dos casos, no período entre outubro de 2020 e março de 2021 . A ficha é utilizada durante os atendimentos aos pacientes, preenchida pelo extensionista/profissional de plantão do CIATOx, no primeiro atendimento ao paciente e durante os próximos dias, com acompanhamento do caso até sua evolução.

Dessa forma, procedeu-se a construção do banco de dados para verificar a ocorrência de possíveis erros de digitação de dados e posterior análise estatística. Assim, a população do estudo compreendeu 301 casos de pacientes com história de ingestão de medicamentos como forma de tentativa de suicídio notificados no SINAN, registrados no período de 2019 , em 
Campina Grande, Paraíba. Foram incluídos na pesquisa pacientes residentes da zona urbana de Campina Grande e excluídos os pacientes cujas fichas estavam com dados incompletos/ausentes totalizando uma amostra final de 237 casos.

Dessa maneira, os registros de casos de tentativas de suicídio por uso de medicamentos e organizados em uma planilha eletrônica utilizando o software Microsoft Office Excel 2013. Para caracterização dos sujeitos do estudo, foram consideradas as variáveis sociodemográficas (sexo, faixa etária, nível de escolaridade, estado civil, situação no mercado de trabalho).

Assim sendo, foi aplicado o teste de Qui-Quadrado $\left(\mathrm{X}^{2}\right)$ de aderência para verificar as diferenças entre as frequências observadas e as frequências esperadas para o perfil socioeconômico dos pacientes que tentaram suicídio.

$\mathrm{Na}$ fase do processamento e análise dos dados, utilizou-se o programa The $R$ Project for Statistical Computing, software com versão 3.4.2. Assim sendo, os dados referentes à frequência dos casos de tentativa de suicídio por ingestão de medicamentos e as características socioeconômicas da população foram demonstradas através de tabelas descritivas, tabelas de frequências absolutas e relativas e testes de correlação para verificar associação entre as variáveis.

Adotou-se como unidade de verificação a variável "bairro de ocorrência” para conduzir a análise espacial da pesquisa. Dessa forma, utilizou-se dados obtidos a partir dos "50 códigos" disponíveis no Departamento Estatística do Centro de Ciências e Tecnologia da Universidade Estadual da Paraíba a fim de construir os mapas do estudo para o município.

Os dados espaciais e a malha digital do município foram captados com auxílio da Secretaria de Planejamento do município, através do Setor de Geoprocessamento.

Na sequência, foi elaborado mapa coroplético com variação de cor a fim de demonstrar a ocorrência e a intensidade do fenômeno. Dessa forma, calculou-se o Índice de Moran Global (I), a fim de observar a dependência espacial dos bairros do município de Campina Grande e o Índice de Moran Local, que possibilita a visualização de cada polígono, demonstrando a auto correlação espacial a partir do Lisa Maps. Adotou-se, para o mapa de Moran, a coloração vermelha, o que permitiu a visualização dos casos de pessoas que tentaram suicídio em determinado bairro. Por outro lado, para construção do Lisa Maps, as cores utilizadas foram: vermelha e azul.

O Lisa Maps categorizou os bairros a partir dos índices locais de Moran e dos valores obtidos, a partir do nível de significância, podendo variar entre -1 e +1 , de acordo com o valor da variável obtida por bairro comparada com a média dos bairros vizinhos: alta/alta ou baixa/baixa, demonstrando autocorrelação espacial positiva, ou seja, vizinhança com valores próximos e alta/baixa ou baixa/alta, demonstrando autocorrelação espacial negativa, ou seja, vizinhança com valores diferentes.

Assim sendo, foi construído o Diagrama de Espalhamento de Moran (Moran Scatterplot) que tem o objetivo de informar os dados necessários para comparação dos valores normalizados do atributo, em cada área, com a média dos seus vizinhos, construindo um gráfico bidimensional de [z] (valores normalizados) por [ $\left.\mathrm{w}_{2}\right]$ (média dos vizinhos), dividido em quatro quadrantes, onde cada ponto representa uma área.

O estudo foi realizado mediante parecer formal do Comitê de Ética em Pesquisa (CEP) da Universidade Estadual da Paraíba (UEPB) sob o número 4.172.667, em harmonia com os princípios e diretrizes apontados pela Resolução Nº 466 de 2012 da CONEP/MS.

\section{Resultados e Discussão}

Foram avaliados 237 pacientes residentes na zona urbana de Campina Grande com relato de tentativa de suicídio através da ingestão de medicamentos. A partir da tabela, 1 é possível identificar que o perfil dos pacientes obedeceu o seguinte padrão: indivíduos do sexo feminino (79,7\%), na faixa etária de 20 a 39 anos (51,5\%), escolaridade em nível de ensino 
fundamental $(49,4 \%)$, condição de solteiro como estado civil $(64,5 \%)$ e a maioria sendo estudante (42,2\%), seguido de desempregado (a) $(32,1 \%)$.

Tabela 1 - Características sociodemográficas dos pacientes que tentaram suicídio através da ingestão de medicamentos, no ano de 2019.

\begin{tabular}{|c|c|c|c|}
\hline Variável & Absoluta (N) & Relativa(\%) & p-valor \\
\hline \multicolumn{4}{|l|}{ Sexo } \\
\hline Feminino & 189 & 79,7 & $<0,001$ \\
\hline Masculino & 48 & 20,3 & \\
\hline \multicolumn{4}{|l|}{ Escolaridade } \\
\hline Ensino Fundamental & 117 & 49,4 & $<0,001$ \\
\hline Ensino Médio & 109 & 46 & \\
\hline Ensino Superior & 11 & 4,6 & \\
\hline \multicolumn{4}{|l|}{ Faixa etária (em anos) } \\
\hline 10-19 anos & 74 & 31,2 & $<0,001$ \\
\hline 20-39 anos & 122 & 51,5 & \\
\hline 40-59 anos & 38 & 16 & \\
\hline$\geq 60$ anos & 3 & 1,3 & \\
\hline \multicolumn{4}{|l|}{ Estado civil } \\
\hline Solteiro (a) & 153 & 64,5 & $<0,001$ \\
\hline Casado (a) & 57 & 24,2 & \\
\hline Divorciado (a) & 25 & 10,5 & \\
\hline Viúvo (a) & 2 & 0,8 & \\
\hline \multicolumn{4}{|l|}{$\begin{array}{l}\text { Situação no mercado de } \\
\text { trabalho }\end{array}$} \\
\hline Estudante & 100 & 42,2 & $<0,001$ \\
\hline Empregado (a) & 33 & 13,9 & \\
\hline Autônomo (a) & 24 & 10,1 & \\
\hline Desempregado (a) & 76 & 32,1 & \\
\hline Aposentado (a) & 4 & 1,7 & \\
\hline
\end{tabular}

Fonte: Autores.

Assim sendo, foi elaborado mapa coroplético a fim de visualizar a distribuição das frequências dos casos de tentativas de suicídio através do uso de medicamentos, de acordo com os bairros do município de Campina Grande. Dessa forma, o mapa foi elaborado com as tonalidades que variaram entre marrom, caracterizando os bairros com maior número de casos, até tons mais claros, como a cor branca, que demonstra menor número de casos, como mostra a Figura 1.

Dessa forma, pode-se perceber ocorrência de casos em praticamente todos os bairros do município, evidenciando-se maior números de casos na zona oeste $(n=92)$, representada pelos bairros Malvinas $(n=23)$, Bodocongó ( $n=21)$ e Serrotão $(n=9)$, seguido da zona sul $(n=70)$, com destaque para os bairros Liberdade $(n=12)$, Catolé ( $n=11)$ e Três Irmãs ( $n=10)$. Por outro lado, os bairros Mirante, Cuités, Sandra Cavalcante, Louzeiro, Itararé e Velame não apresentaram ocorrências de casos de tentativas de suicídio através do uso de medicamentos. 
Figura 1 - Mapa de quartil relativo às frequências observadas dos casos de tentativas de suicídio na zona urbana de Campina Grande, Paraíba, para o ano de 2019.

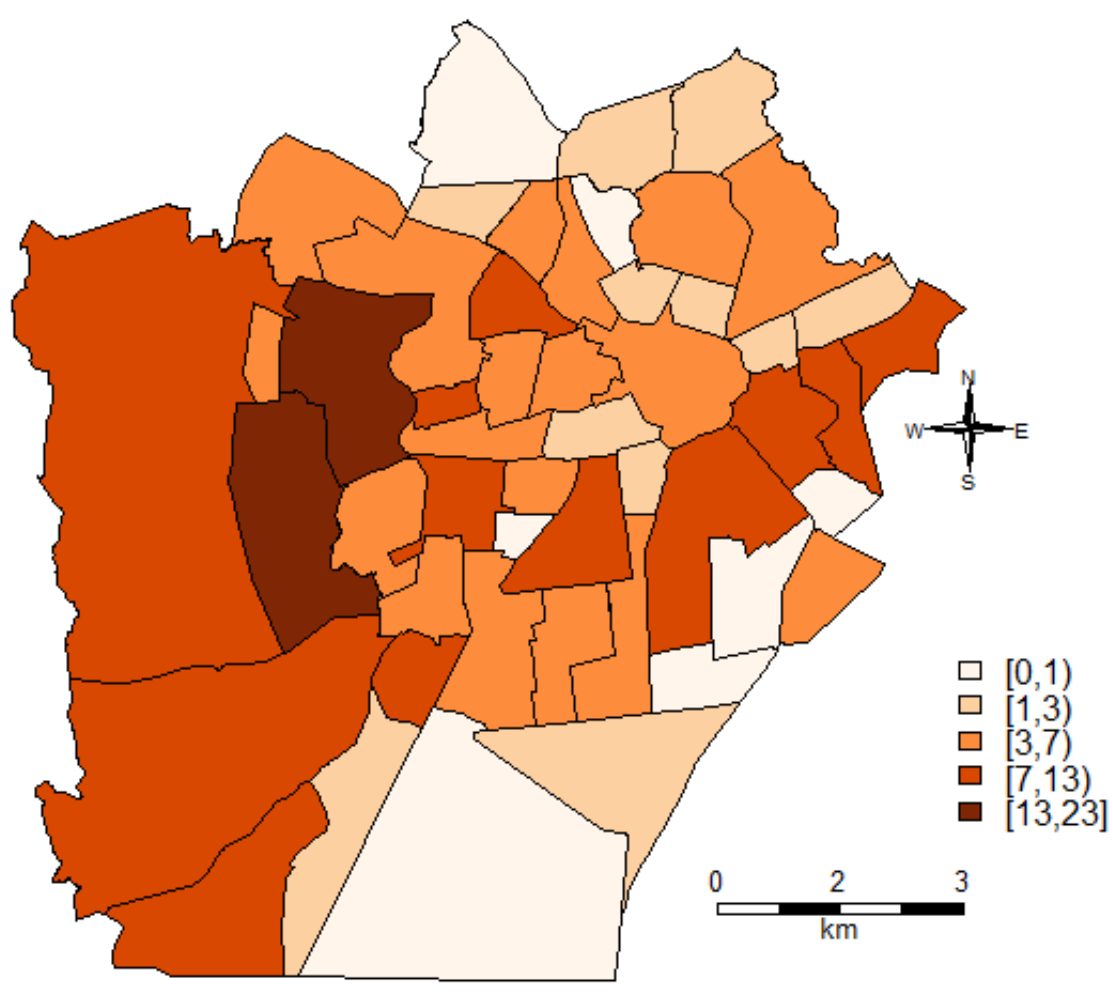

Fonte: Autores.

Então, calculou-se o Índice de Moran Global (I) a fim de observar a interdependência espacial dos bairros do município de Campina Grande e, de forma complementar, o Índice de Moran Local (Local Indicators of Spatial Association Lisa). Com isso, a fim de facilitar a observação da similaridade dos valores do atributo das áreas em estudo, foram construídos os seguintes mapas: Lisa Map, Mapa de espalhamento de Moran e Moran Map.

Assim, verificou-se que, no quesito global, no ano de 2019, não houve autocorrelação espacial na região estudada, com Índice de Moran Global encontrado de 0,1210 (Tabela 2), mostrando que o fenômeno aconteceu de forma independente.

Tabela 2 - Avaliação da autocorrelação espacial por meio do Índice de Moran Global (I) para os bairros do município de Campina Grande, no ano de 2019.

\begin{tabular}{ccc}
\hline Variável & I de Moran & $p$-valor \\
\hline Ano de 2019 & 0,1210 & 0,04373 \\
\hline
\end{tabular}

Fonte: Autores.

Dessa maneira, utilizando-se o Diagrama de espalhamento de Moran (ou Moran Scatterplot), verificam-se os bairros que apresentam dependência com os bairros vizinhos, o que demonstra capacidade de influenciar nas características da vizinhança. A partir da Figura 2, observa-se a maneira a qual se comportam determinados bairros de Campina Grande de acordo com a residência das pessoas do estudo. Desse modo, os bairros que apresentaram significância espacial foram: Bodocongó (24), Malvinas (26) e, mais discretamente, Dinamérica (27).

Sendo assim, nota-se o comportamento de determinados bairros do município de Campina Grande relativo à 
residência das pessoas que tentaram suicídio através do uso de medicamentos. Dessa forma, entende-se que houve uma predominância de bairros nos quadrantes Q2 e Q4. Além disso, observou-se, em Q1 e Q2, bairros com associação espacial positiva, ou seja, uma localidade possui vizinhos com valores parecidos, enquanto Q3 e Q4 apresentam autocorrelação negativa, ou seja, há dissimilaridade entre as áreas vizinhas.

Figura 2 - Diagrama de espalhamento de Moran para os casos de tentativas de suicídio por uso de medicamentos, no município de Campina Grande, Paraíba, Brasil, no ano de 2019.

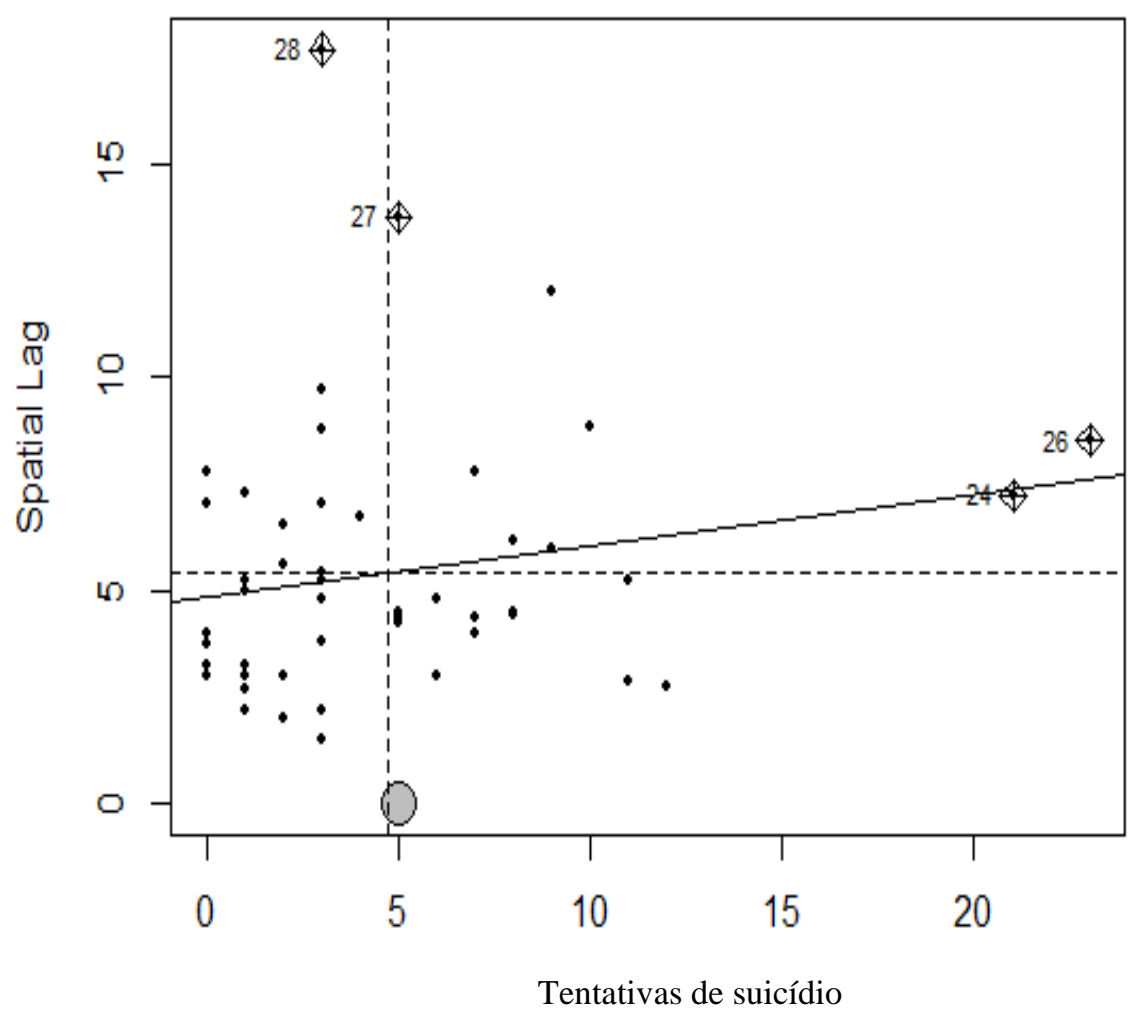

Fonte: Autores.

Por outro lado, a fim de observar a autocorrelação espacial local, foram construídos mapas temáticos do tipo Lisa, que possibilita a visualização de cada polígono, evidenciando o respectivo quadrante no gráfico de espalhamento. Assim sendo, as cores vermelha e azul indicam áreas de influência espacialmente significativas, enquanto a cor branca evidencia a "não significância”, ou seja, a não correlação espacial.

Dessa maneira, a partir do Lisa Map, é possível observar que os bairros que apresentaram maior significância estatística foram: Serrotão, Malvinas, Bodocongó e Três Irmãs (Figura 3). 
Figura 3 - Indicador Local de Autocorrelação espacial (Lisa) para a variável bairros onde residem indivíduos que tentaram suicídio por meio do uso de medicamentos, no município de Campina Grande, no ano de 2019.

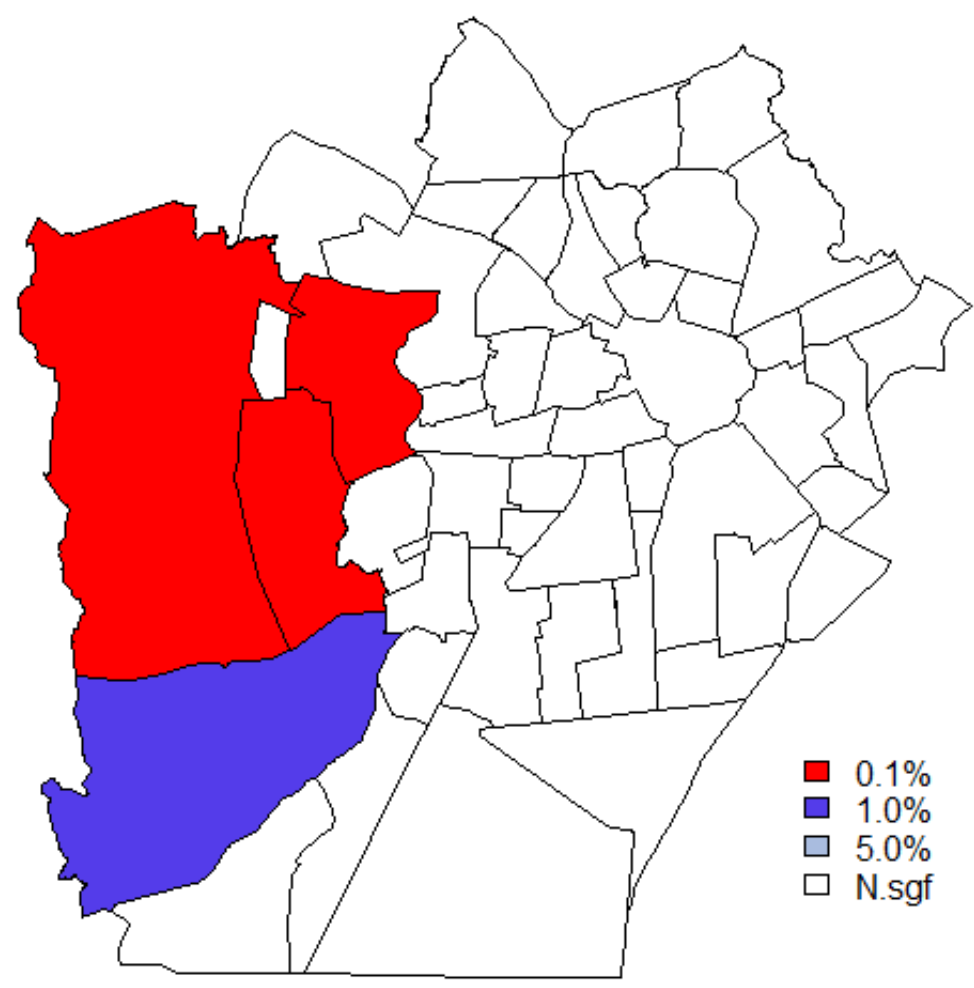

Fonte: Autores.

\section{Discussão}

Nos últimos anos, cresceu consideravelmente o número de casos de violência, principalmente em grandes centros urbanos, o que contribuiu para maior enfoque das políticas públicas em todo o mundo acerca da problemática (Bezerra Filho, Werneck, Almeida, Oliveira \& Magalhães, 2012). Dentre as diversas causas de violência, encontra-se o suicídio e as tentativas de suicídio, eventos complexos e multicausais (Who, 2016).

O perfil das pessoas que tentaram suicídio no período citado através do uso de medicamentos não difere do encontrado em outras pesquisas realizadas no Brasil (da Silva et al., 2021; Rosa, Agnolo, Oliveira, Mathias \& Oliveira, 2016; Ribeiro, Castro, Scatena \& Haas, 2018; Félix et al., 2016). Em 2014, a incidência nacional na população feminina em relação à ingestão de medicamentos, mostrou que o sexo feminino cometeu mais tentativa de suicídio, com 10,3 casos (100.000 hab./ano) quando comparado com o sexo masculino, que cometeu 2,0 tentativas de autoextermínio (100.000 hab./ano) (Fernandes et al., 2021; Ribeiro et al., 2018; Félix et al., 2016).

O uso de medicamentos tem destaque considerável nas tentativas de suicídio, sendo a forma mais comum relacionada a este tipo de agravo (Alves et al., 2021). Estudo realizado entre 2013 e 2014 no Ceará também indicou que a maioria das tentativas de suicídio no Estado ocorreu através do uso de medicamentos (Oliveira et al., 2015).

Dessa maneira, quanto ao método utilizado pelas mulheres para as tentativas de suicídio, nota-se que os mais utilizados foram são os medicamentos, achado que está em conformidade com os resultados da literatura (Sereno, Silva \& da Silva, 2020; Monteiro, Bahia, Paiva, Sá \& Minayo, 2015; Vieira, Santana \& Suchara, 2015). Alguns autores acreditam que essa ocorrência deve-se ao fato de que a mulher procura formas mais brandas e menos invasivas de tentativa de suicídio quando comparadas aos homens (Brasil, 2019; Vidal, Gontijo \& Lima, 2013). 
Assim, percebe-se um perfil de tentativas de suicídio por medicamentos predominantemente composto por mulheres $(79,7 \%)$. Desse modo, entende-se que as diferenças de gênero relacionadas às tentativas de suicídio através do uso de medicamentos podem ser explicadas por fatores de natureza econômica, social e cultural (Fernandes et al., 2021; Meneghel, Moura, Hesler \& Gutierrez, 2015). Ao mesmo tempo que a mulher conseguiu espaço no mercado de trabalho, observa-se, nos dias atuais, um percentual elevado de casos entre mulheres que não trabalham fora de casa, demonstrando a importância de um trabalho fora de casa para a saúde mental das pessoas (Vidal, Gontijo \& Lima, 2013; Cavalcante \& Minayo, 2012; Rangel \& Francilino, 2018).

No quesito idade, nota-se que a maioria das pessoas do estudo apresentou um perfil adulto, jovem, com idade entre 20 e $39 \operatorname{anos}(51,5 \%)$, dados que corroboram com a literatura (da Silva et al., 2021; Machado \& Santos, 2015; da Silva Miranda et al., 2020). Esses comportamentos apresentados em tal idade envolvem questões complexas relacionadas à problemas familiares e conjugais, busca pela inserção no mercado de trabalho, problemas emocionais, psiquiátricos, abuso na infância (Graner \& Cerqueira, 2019; Bahia, Avanci, Pinto \& Minayo, 2017). Além disso, percebe-se a alta participação de adolescentes e jovens na faixa etária de 10 a 19 anos nas tentativas de suicídios (31,2\%), visto que essa fase é considerada bastante estressante devido às mudanças hormonais, comportamentais, físicas e psicológicas típicas dessa idade (Claumann, Pinto, Silva \& Pelegrini, 2018; Vidal, Gontijo \& Lima, 2013), além de insegurança em relação ao futuro, cobranças da família e transição da fase da adolescência para a vida adulta (Gomes \& da Silva, 2020).

Entre os fatores considerados de risco para as tentativas de suicídio, encontra-se a baixa escolaridade, achado que está em conformidade com estudos da literatura, que sugerem que quanto menor o grau de instrução, maior o risco de ideação suicida (Vidal, Gontijo \& Lima, 2013; Vasconcelos-Raposo, Soares, Silva, Fernandes \& Teixeira, 2016; Carvalho et al., 2020). Acredita-se que esta ocorrência deve-se ao fato de que pessoas com baixo nível de escolaridade tende a ter mais dificuldade de inserção no mercado de trabalho e, consequentemente, em ter independência financeira, além de aumentar a insegurança profissional e pessoal (Carvalho et al., 2020; Zhou, Wang, Jia \& Ma, 2019).

De forma similar, as pessoas do estudo apresentaram situação socioeconômica frágil, visto que a maioria era estudante $(42,2 \%)$, seguido por pessoas que encontravam-se desempregadas $(32,1 \%)$, corroborando com estudos que veem o fato da pessoa estar empregada como um fator preventivo para a ideação suicida (Cavalcante \& Minayo, 2012; Vasconcelos-Raposo et al., 2016).

Quanto ao estado civil, verificou-se que os solteiros constituíram a maioria (64,5\%), dado que se assemelha com a alta prevalência de tentativas de suicídio entre solteiros (Carvalho et al., 2020). Um estudo realizado em 15 municípios de Minas Gerais demonstrou que as tentativas de suicídio eram cometidas, com maior frequência, por pessoas solteiras e jovens, evidenciando maior risco de para este tipo de evento em pessoas que não possuíam companheiros (Vidal, Gontijo \& Lima, 2013).

Porém, vale salientar que a amostra do presente estudo é composta por população predominantemente jovem, o que pode explicar a ocorrência de pessoas solteiras em estudo.

A partir do uso de Sistemas de Informações Geográficas (SIG) e de técnicas de geoprocessamento, é possível identificar e mapear áreas sob maior risco de agravo, além de permitir observar a distribuição espacial dos casos de tentativas de suicídio por meio do uso de medicamentos e alocar recursos de Saúde Pública eficientes (Sousa \& Pinto, 2016).

No que diz respeito à Análise espacial, no ano de 2019, os casos de tentativas de suicídio através do uso de medicamentos ocorreram em praticamente todos os bairros do município, com ênfase para os bairros Malvinas, Bodocongó e Liberdade, que apresentaram os maiores índices de residências da população estudada. Outrora, vale salientar que os bairros Mirante, Cuités, Sandra Cavalcante, Louzeiro, Itararé e Velame consistem em bairros sem nenhum registro de casos no ano analisado neste estudo, fato que suscita a dúvida em relação à subnotificação dos casos de tentativas de suicídio no município, 
muitas vezes ocasionada pela recusa da pessoa em procurar o serviço de saúde de referência. Lamentavelmente, muitos casos de tentativas de suicídio não chegam ao serviço de saúde de referência para correta notificação do agravo, muitas vezes por serem de baixa complexidade.

Em relação à autocorrelação espacial, adotou-se a variável "bairro de residência" das pessoas que tentaram suicídio através do uso de medicamentos. Dessa forma, buscou-se estabelecer a relação existente entre os bairros e o poder de influência que um possuía sobre seus vizinhos através do cálculo do Índice Global de Moran e Índice Local de Moran.

Com base na metodologia utilizada, não observou-se autocorrelação espacial significativa de acordo com o modelo do Índice de Moran Global para o ano de 2019. Por outro lado, através do Índice de Moran Local, observou-se que alguns bairros apresentaram influência na vizinhança. Dessa maneira, os bairros que apresentaram autocorrelação espacial positiva, localmente, foram: Serrotão, Malvinas, Bodocongó e Três Irmãs. Os três primeiros bairros (Serrotão, Malvinas e Bodocongó) pertencem à zona oeste do município, enquanto o último (Três Irmãs) pertence à zona sul, todos localizados em bairros periféricos da cidade.

Dessa maneira, ressalta-se a importância de conhecer o comportamento das Tentativas de Suicídio para contribuir com informações até então desconhecidas sobre o padrão espacial do agravo e identificação de áreas vulneráveis que necessitam de intervenção. Além disso, acrescenta-se a isso a necessidade de desenvolvimento e incentivo a novas pesquisas que possam auxiliar na prevenção da problemática em estudo.

\section{Conclusão}

A partir deste estudo, percebe-se que as tentativas de suicídio são um grave problema de saúde pública mundial, que podem acarretar consequências severas, dentre elas o próprio suicídio. Por causa disso, percebeu-se a necessidade de investigar o cenário das tentativas de suicídio no município através do uso de medicamentos no município em questão.

Os dados apresentados permitiram descrever o perfil da população que tenta suicídio através do uso de medicamentos: população predominantemente jovem, com maior prevalência entre a faixa etária de 20 a 39 anos, sexo feminino, com baixo nível de escolaridade e baixa renda.

Além disso, este estudo permitiu demonstrar que a análise espacial é um importante instrumento para monitorização dos casos de tentativas de suicídio, que auxilia na elaboração de estratégias para controle do agravo.

Acerca da estatística espacial, percebeu-se o maior número de casos registrados na zona oeste na cidade, representada pelos bairros Malvinas, Bodocongó e Serrotão; seguido da zona sul, representada pelos bairros Liberdade, Catolé e Três Irmãs. Por outro lado, os bairros Mirante, Sandra Cavalcante, Cuités, Louzeiro, Itararé e Velame não apresentaram casos de tentativas de suicídio por medicamentos no período estudado.

Diante do exposto, destaca-se que os casos de tentativas de suicídio através do uso de medicamentos no município de Campina Grande apresentaram significância estatística quanto à autocorrelação espacial. Verificou-se, também, a autocorrelação espacial local positiva para quatro bairros do município.

Assim, nota-se que o estudou mostrou-se relevante, com contribuição significativa no fortalecimento do controle das tentativas de suicídio, assim como no entendimento do fenômeno que permite a identificação de áreas consideráveis de maior risco para o surgimento deste tipo de agravo. O estudo torna-se um instrumento facilitador para elaboração de medidas eficazes que contribuam para a adoção de políticas públicas sobre tentativas de suicídio e melhorias nos determinantes sociais da saúde relacionados às tentativas de suicídio.

Tendo em vista o que foi apresentado, o presente trabalho irá contribuir para nortear pesquisas futuras e para dar respaldo científico para esses novos estudos, para pesquisas de campo, com uso de metodologias que permitam a discussão da 
temática, assim como a proximidade com as pessoas participantes do estudo, a fim de obter informações diretamente da população que tenta suicídio.

\section{Referências}

Alves, A. K. R., da Silva, B. B. L., de Almeida, B. C., de Brito Pereira, R., dos Santos Silva, L., Alves, A. K. R., \& de Farias, D. R. (2021). Análise do perfil epidemiológico das intoxicações exógenas por medicamentos no Piauí, 2007 a 2019. Research, Society and Development, 10(12), e197101220255e197101220255.

Bahia, C. A., Avanci, J. Q., Pinto, L. W., \& Minayo, M. C. D. S. (2017). Lesão autoprovocada em todos os ciclos da vida: perfil das vítimas em serviços de urgência e emergência de capitais do Brasil. Ciência \& Saúde Coletiva, 22, 2841-2850.

Bezerra Filho, J. G., Werneck, G. L., Almeida, R. L. F. D., Oliveira, M. I. V. D., \& Magalhães, F. B. (2012). Estudo ecológico sobre os possíveis determinantes socioeconômicos, demográficos e fisiográficos do suicídio no Estado do Rio de Janeiro, Brasil, 1998-2002. Cadernos de Saúde Pública, 28, 833-844.

Brasil (2011). Portaria GM/MS Nº 104, de 25 de janeiro de 2011. Ministério da Saúde.

Brasil (2014). Portaria n ${ }^{\circ}$ 1.271, de 06 de junho de 2014. Ministério da Saúde.

Brasil (2019). Boletim Epidemiológico v. 50, $\mathrm{n}^{\circ}$ 24: Perfil epidemiológico dos casos notificados de violência autoprovocada e óbitos por suicídio entre jovens de 15 a 29 anos no Brasil, 2011 a 2018. Ministério da Saúde.

Carvalho, M. L. D. et al. (2020). Suicídio em idosos: abordagem dos determinantes sociais da saúde no modelo de Dahlgren e Whitehead. Revista Brasileira de Enfermagem, 73.

Cavalcante, F. G., \& Minayo, M. C. D. S. (2012). Autópsias psicológicas e psicossociais de idosos que morreram por suicídio no Brasil. Ciência \& saúde coletiva, 17, 1943-1954.

Claumann, G. S., Pinto, A. D. A., Silva, D. A. S., \& Pelegrini, A. (2018). Prevalência de pensamentos e comportamentos suicidas e associação com a insatisfação corporal em adolescentes. Jornal Brasileiro de Psiquiatria, 67, 3-9.

da Silva Miranda, C. C. et al. (2020). Perfil epidemiológico dos casos notificados de tentativas de suicídio por intoxicação exógena no estado do Piauí. Research, Society and Development, 9(9), e798997862-e798997862.

da Silva, A. K. M., da Silva Costa, M. F., Vaz, J. L. S., da Silva Souza, K. A., da Cruz, L. P. L., Freitas, J. E. D. S. M., \& de Oliveira, E. H. (2021). Análise de intoxicações exógenas no Estado do Piauí no período de 2013 a 2017. Research, Society and Development, 10(10), e505101017260-e505101017260..

Félix, T. A. et al. (2016). Fatores de risco para tentativa de suicídio: produção de conhecimento no Brasil. Revista Contexto \& Saúde, $16(31), 173-185$.

Fernandes, T. C. et al. (2021). Intoxicação medicamentosa no estado do Maranhão: uma análise dos casos notificados entre os anos de 2010 a 2020. Research, Society and Development, 10(14), e147101421672-e147101421672.

Graner, K. M., \& Cerqueira, A. T. D. A. R. (2019). Revisão integrativa: sofrimento psíquico em estudantes universitários e fatores associados. Ciência \& Saúde Coletiva, 24, 1327-1346.

Gomes, C. F. M., \& da Silva, D. A. (2020). Aspectos epidemiológicos do comportamento suicida em estudantes universitários. Research, Society and Development, 9(5), e38953106-e38953106.

Klinger, E. I. et al. (2016). Intoxicação exógena por medicamentos na população jovem do Rio Grande do Sul. Rev Epidemiol Controle Infecç, 6(Supl 2), 1-8.

Machado, D. B., \& Santos, D. N. D. (2015). Suicídio no Brasil, de 2000 a 2012. Jornal Brasileiro de Psiquiatria, 64, 45-54.

Magalhães, M. D. A. F. M., \& Medronho, R. D. A. (2017). Análise espacial da Tuberculose no Rio de Janeiro no período de 2005 a 2008 e fatores socioeconômicos associados utilizando microdado e modelos de regressão espaciais globais. Ciência \& Saúde Coletiva, 22, 831-840.

Maior, M. D. C. L. S., Osorio-de-Castro, C. G. S., \& Andrade, C. L. T. D. (2017). Internações por intoxicações medicamentosas em crianças menores de cinco anos no Brasil, 2003-2012. Epidemiologia e Serviços de Saúde, 26, 771-782.

Meneghel, S. N., Moura, R., Hesler, L. Z., \& Gutierrez, D. M. D. (2015). Tentativa de suicídio em mulheres idosas-uma perspectiva de gênero. Ciência \& Saúde Coletiva, 20, 1721-1730.

Monteiro, R. A., Bahia, C. A., Paiva, E. A., Sá, N. N. B. D., \& Minayo, M. C. D. S. (2015). Hospitalizações relacionadas a lesões autoprovocadas intencionalmente-Brasil, 2002 a 2013. Ciência \& Saúde Coletiva, 20, 689-699.

Moreira, D. L., Martins, M. C., do Amaral Gubert, F., \& de Sousa, F. S. P. (2015). Perfil de pacientes atendidos por tentativa de suicídio em um centro de assistência toxicológica. Ciencia y Enfermeria, 21(2), 63-75.

Mota, D. M. et al. (2012). Perfil da mortalidade por intoxicação com medicamentos no Brasil, 1996-2005: retrato de uma década. Ciência \& Saúde Coletiva, 17, 61-70.

Oliveira, E. N. et al. (2015). Tentativa de suicídio por intoxicação exógena: contexto de notificações compulsórias. Revista Eletrônica Gestão e Saúde, (3), 2497-2511. 
Pereira, A. S., et al. (2018). Metodologia do trabalho científico. UFSM.

Rangel, N. L., \& Francelino, E. V. (2018). Caracterização do Perfil das Intoxicações Medicamentosas no Brasil, durante 2013 a 2016. Id on Line Revista De Psicologia, 12(42), 121-135.

Ribeiro, N. M., Castro, S. D. S., Scatena, L. M., \& Haas, V. J. (2018). Análise Da Tendência Temporal Do Suicídio E De Sistemas De Informações Em Saúde Em Relação Às Tentativas De Suicídio. 1. Texto \& Contexto-Enfermagem, 27.

Rosa, N. M. D., Agnolo, C. M. D., Oliveira, R. R. D., Mathias, T. A. D. F., \& Oliveira, M. L. F. D. (2016). Tentativas de suicídio e suicídios na atenção préhospitalar. Jornal Brasileiro de Psiquiatria, 65, 231-238.

Sereno, V. M. B., Silva, A. S., \& da Silva, G. C. (2020). Perfil epidemiológico das intoxicações por medicamentos no Brasil entre os anos de 2013 a 2017. Brazilian Journal of Development, 6(6), 33892-33903.-135.

Sousa, A. I. A. D., \& Pinto, V. L. (2016). Análise espacial e temporal dos casos de aids no Brasil em 1996-2011: áreas de risco aumentado ao longo do tempo. Epidemiologia e Serviços de Saúde, 25, 467-476.

Sinan. (2021). Sistema de Informação de Agravos de Notificação. http://www.portalsinan.saude.gov.br

Timóteo, M. V. F. et al. (2020). Panorama das intoxicações associadas ao uso de medicamentos registradas no Brasil. Research, Society and Development, 9(4), e142942993-e142942993.

Vasconcelos-Raposo, J., Soares, A. R., Silva, F., Fernandes, M. G., \& Teixeira, C. M. (2016). Níveis de ideação suicida em jovens adultos. Estudos de Psicologia (campinas), 33, 345-354.

Vidal, C. E. L., Gontijo, E. C. D. M., \& Lima, L. A. (2013). Tentativas de suicidio: factores pronósticos y estimativa del exceso de mortalidad. Cadernos de saúde publica, 29(1), 175-187

Vieira, L. P., Santana, V. T. P. D., \& Suchara, E. A. (2015). Caracterização de tentativas de suicídios por substâncias exógenas. Cadernos Saúde Coletiva, 23, $118-123$.

World Health Organization. (2016). Suicide rate per 100,000 population by WHO region. https:/www.who.int/mental_health/suicide prevention/per_100.000_population_2016.JPG?ua=1

World Health Organization. (2019). Suicide in the world: global health estimates (No. WHO/MSD/MER/19.3). World Health Organization.

Zhou, L., Wang, G., Jia, C., \& Ma, Z. (2019). Being left-behind, mental disorder, and elderly suicide in rural China: a case-control psychological autopsy study. Psychological medicine, 49(3), 458-464. 